\title{
Use of photoplethysmography to predict mortality in intensive care units
}

This article was published in the following Dove Press journal: Vascular Health and Risk Management

\section{Kelser de Souza Kock' Jefferson Luiz Brum Marques $^{2}$ \\ 'Graduate Program in Medical Sciences, Federal University of Santa Catarina, Florianópolis, SC. Brazil; ' Institute of Biomedical Engineering, Department of Electrical and Electronic Engineering, Federal University of Santa Catarina, Florianópolis, SC, Brazil}

\section{Video abstract}

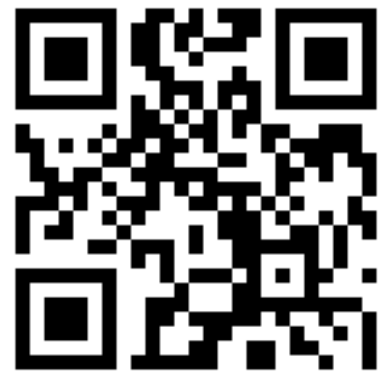

Point your SmartPhone at the code above. If you have a QR code reader the video abstract will appear. Or use: http://youtu.be/caD5ce3VfrYO
Correspondence: Kelser de Souza Kock Graduate Program in Medical Sciences, Federal University of Santa Catarina, Galdino José de Bessa, 164, Oficinas, Tubarão, SC 88702-220, Brazil Tel +55489999698II

Email kelserkock@yahoo.com.br
Purpose: The aim of this study was to evaluate and compare the capacity to predict hemodynamic variables obtained with photoplethysmography (PPG) and Acute Physiology and Chronic Health Evaluation (APACHE II) in patients hospitalized in the intensive care unit (ICU).

Materials and methods: A prospective cohort study was conducted in the adult ICU of Hospital Nossa Senhora da Conceição, located in Tubarão, Santa Catarina, Brazil. The data collected included the diagnosis for hospitalization, age, gender, clinical or surgical profile, PPG pulse curve signal, and APACHE II score in the first 24 hours. A bivariate and a multivariate logistic regressions were performed, with death as an outcome. A mortality model using artificial neural networks (ANNs) was proposed.

Results: A total of 190 individuals were evaluated. Most of them were males (6:5), with a median age of 67 (54-75) years, and the main reasons for hospitalization were cardiovascular and neurological causes; half of them were surgical cases. APACHE II median score was 14 (8-19), with a median length of stay of $6(3-15)$ days, and $28.4 \%$ of the patients died. The following factors were associated with mortality: age (OR=1.023; 95\% CI 1.001-1.044; $P=0.039)$, clinical profile $(\mathrm{OR}=5.481 ; 95 \%$ CI 2.646-11.354; $P<0.001)$, APACHE II $(\mathrm{OR}=1.168 ; 95 \%$ CI 1.106-1.234; $P<0.001)$, heart rate in the first 24 hours $(\mathrm{OR}=1.020 ; 95 \%$ CI 1.001-1.039; $P=0.036)$, and time between the systolic and diastolic peak $(\Delta T)$ intervals obtained with PPG $(\mathrm{OR}=0.989 ; 95 \%$ CI $0.979-0.998 ; P=0.015)$. Compared with the accuracy (area under the receiver-operating characteristic curve) 0.780 of APACHE II ( $95 \%$ CI $0.711-0.849 ; P<0.001)$, the multivariate logistic model showed a larger area of $0.858(95 \%$ CI $0.803-0.914 ; P<0.001)$. In the model using ANNs, the accuracy was 0.895 (95\% CI 0.851-0.940; $P<0.001$ ).

Conclusion: The mortality models using variables obtained with PPG, with the inclusion of epidemiological parameters, are very accurate and, if associated to APACHE II, improve prognostic accuracy. The use of ANN was even more accurate, indicating that this tool is important to help in the clinical judgment of the intensivist.

Keywords: photoplethysmography, intensive care units, computer-aided signal processing, pulse wave analysis, prognosis, hemodynamic monitoring

\section{Introduction}

Intensive care units (ICUs) are characterized by high complexity, multi/interdisciplinarity, and state-of-the-art technology. Such a profile represents an ideal place to take care of seriously ill patients. ${ }^{1}$

The first form of screening to classify the severity of an illness and intensive care was carried out by Florence Nightingale in 1863. Since then, this evaluation had been made in a subjective manner by clinical judgment, until the development of the Acute 
Physiology and Chronic Health Evaluation score (APACHE) in 1981, which allowed an objective evaluation of severity. ${ }^{2}$

Nowadays, score systems are routinely used in ICUs to predict the end point or to evaluate the severity of organic dysfunction. Such scales generally use clinical and laboratory data to reach a score. The most common systems are APACHE II, Simplified Acute Physiology Score, Multiple Organ Dysfunction Score, and Sequential Organ Failure Assessment. However, in some situations, they are not very accurate, as in the subgroups of patients or in specific diseases. Thus, other scores are important to help in the clinical evaluation of seriously ill patients. ${ }^{3}$

In this perspective, noninvasive measures to help stabilizing the prognosis are important, as their use is not expensive and represents minimal risk. Photoplethysmography (PPG) is an optical noninvasive technique that monitors the pulse wave, capturing the variation of the peripheral blood volume, by means of the Beer-Lambert law. The basis of this theory is that the absorption of light is directly related to the wavelength of the light source and the concentration of substances in the optical path. The first description of PPG occurred in 1937 by Hertzman and Spealman. Later, the AC (alternating coupling, corresponding the pulsatile arterial blood flow) and DC (direct coupling, related to the tissue background and venous blood) components and their interaction with red and infrared light were identified, leading to the development of pulse oximeters. However, it works by means of a light beam, and situations such as hypothermia, severe hypotension, and motor agitation can reduce the signal-to-noise ratio. ${ }^{4}$

In addition to the information included in the peripheral pulse oximetry, such as the heart rate (HR) and peripheral oxygen saturation $\left(\mathrm{SpO}_{2}\right)$, other relevant parameters are present in the PPG signal, even though they are commonly underused. Besides HR, the PPG signal is influenced by hemodynamic status, physiological condition, and arteriolar properties. In this sense, studies have shown that variables of cardiovascular function, such as arterial stiffness, peripheral resistance, tissue perfusion, hypovolemia, and sympathovagal balance, are present in the photoplethysmographic pulse wave. ${ }^{4-7}$

For these purposes, the evaluation of the pulse curve variation requires a nontrivial analysis, requiring differential calculus for the composition of the first and second derivatives and identification of the regions of interest. This mathematical manipulation requires the use of computational algorithms that allow the identification of the characteristics of the pulse wave, such as systolic peak (SP) and diastolic peak (DP). ${ }^{8}$ Among the parameters used, the time interval between SP and DP is associated with the reflection of the pulse wave at the distal vascular bed, representing arterial stiffness. ${ }^{9}$ In addition to the time interval between SP and DP, the proportion of the amplitude of these peaks is also related to the arterial compliance and total peripheral resistance. Another parameter obtained by the PPG, analyzing its second derivative is called the PPG acceleration. This can provide information about the blood flow acceleration. The relationship between these peaks indicates the state of arterial aging. ${ }^{10}$ Still, from the analysis of the components AC and DC and oscillation of the SP amplitude, the perfusion index (PI) and PPG variability can be obtained. These parameters refer to peripheral perfusion ${ }^{11}$ and volume status, respectively, indicating responsiveness to fluid therapy infusion. ${ }^{12}$ Also, evaluating the time intervals between SPs, a beat-to-beat temporal analysis, inferred the state of the autonomic nervous system. ${ }^{13}$ Therefore, the purpose of this study was to evaluate and compare the capacity to predict hemodynamic variables obtained from PPG and APACHE II combined analysis in ICU hospitalized patients by means of logistic regression and artificial neural networks (ANNs). Besides avoiding invasiveness, the cardiovascular variables identified through this technique improve the clinical judgment in intensive care setting.

Some researches compare risk prediction in medicine using ANNs and logistic regression. The results seem to be better with ANNs, which has the advantage to incorporate nonlinear effects. Difficulty in interpreting the model parameters is a disadvantage, despite that both techniques prove important as predictive tools. ${ }^{14,15}$

\section{Materials and methods}

A prospective cohort study was conducted in the adult intensive care center (ICC) of Hospital Nossa Senhora da Conceição, located in Tubarão, Santa Catarina, Brazil. This ICC has three ICUs, each one with 10 beds, serving an area of 18 municipalities. One of the ICUs is intended to treat patients with cardiovascular diseases and the other two have a general care profile.

Individuals hospitalized from July 2016 to July 2017 were selected, whose relatives have signed the voluntary informed consent form. The poor quality of PPG signal and the individuals transferred from the ICUs were considered as exclusion criteria. This study was submitted to the research ethics committee of the University of Southern Santa Catarina (UNISUL), located in Palhoça, SC, Brazil, and was approved by CAAE 50687515.6.0000.5369.

As this is an accuracy study, for sample size calculation, a mortality prevalence of $26 \%(P=0.26)$ was considered, in accordance with a previous estimate, with an error of $10 \%$ 
$(e=0.1)$ in the CI of $95 \%\left(Z_{\alpha / 2}=1.96\right)$. The sensitivity was determined as $85 \%$ (Sens $=0.85$ ). According to Equation 1, the sample size obtained was 188 patients: ${ }^{16}$

$$
n_{\text {sens }}=\frac{\left(Z_{\alpha / 2}\right)^{2} \text { Sens. }(1-\text { Sens })}{e^{2} \cdot P}
$$

In the data collection, we included the diagnosis for hospitalization, age, gender, PPG pulse curve signal, clinical or surgical profile, and APACHE II score, ${ }^{17}$ in the first 24 hours. The individual was followed up until the end point (hospital discharge or death), including the time of hospitalization in the ICU.

For acquisition of the PPG signal, a Reflex Aqwave ${ }^{\mathrm{TM}}$ oximeter was used. It can store the pulse curve values in a sampling frequency of $60 \mathrm{~Hz}$ and duration of 1 minute. The device can export the text file data for computational analysis. The data from the PPG pulse curve were manipulated in the MATLAB software. Initially, the PPG raw signal processing was performed by a low-pass filter of $12 \mathrm{~Hz}$ to extract the noise. After smoothing, the first and second derivatives of PPG were made. Peaks, valleys, and time between peaks were identified. The values below and above the interquartile distance were excluded. The medians of the PPG interest variables were selected for analysis.

The variables related to cardiovascular function, arterial stiffness, and peripheral resistance were crest time (CT), time between the SP and DP $(\Delta T)$, augmented index resulting from the division of the DP and SP, and arterial aging, obtained with the second derivative of the PPG signal. ${ }^{7}$ For the analysis of peripheral perfusion and volemia, the expressions PI and pleth variability index $(\mathrm{PVI})^{5}$ were used, and to evaluate the autonomic nervous system, the root mean square of the differences between the adjacent SPs (rMSSD) ${ }^{18}$ were used. Equations 2-6 describe the calculation of these parameters:

$$
\begin{gathered}
\mathrm{AI}=100 \% *[\mathrm{PD} / \mathrm{PS}] \\
\text { Arterial Aging }=100 \% \times[\mathrm{b} / \mathrm{a}] \\
\mathrm{PI}=100 \% \times[\mathrm{AC} / \mathrm{DC}] \\
\mathrm{PVI}=\left[\left(\mathrm{SP}_{\text {max }}-\mathrm{SP}_{\min }\right) / \mathrm{SP}_{\max }\right] \times 100 \% \\
\mathrm{rMSSD}=\sqrt{\frac{1}{N-1} \sum_{i-1}^{N-1}\left((\mathrm{PS}-\mathrm{PS})_{i+1}-(\mathrm{PS}-\mathrm{PS})_{i}\right)^{2}}
\end{gathered}
$$

The identification of the PPG parameters is described in Figure 1.
The data were stored in a database created with the assistance of the Excel ${ }^{\circledR}$ software and then exported to the SPSS $20.0^{\circledR}$ software. The data were presented in absolute numbers and percentages and measures of central tendency and dispersion. The variables were compared in relation to the end point (hospital discharge or death) by means of gross and adjusted logistic regression. For multivariate analysis, the backward method was used, and it was completed only with significant variables $(\alpha=5 \%)$. The variables associated to the end point were selected for training (70\%) and test (30\%) by the ANN multilayer perceptron. ${ }^{19}$ The accuracy of the multivariate models was compared with APACHE II using the receiver-operating characteristic (ROC) curve. To compare the ROC curves, the DeLong method was used. ${ }^{20}$

\section{Results}

A total of 230 individuals participating in the research were followed up, of which 14 were excluded for poor quality of signal and 26 for error in the medical record identification, totaling 190 patients. The median (p25-p75) age was 67 (54-75) years, with higher prevalence of men and the most common diagnoses for hospitalization in the ICU were cardiovascular and neurological causes, with more than half of the cases being surgical. The characteristics of the sample are described in Table 1.

Figure 2 shows the histogram of the variables obtained with the PPG signal, identifying the mean \pm SD and median (p25-p75).

In the bivariate analysis, the variables related to death were age, surgical, APACHE II, time of hospitalization, HR, and $\Delta T$ (Table 2).

By using some PPG variables and an epidemiological variable, three models for mortality prediction in ICUs were developed. In the comparison of Models 1, 2, and 3 with APACHE II, there was no statistical difference $(P=0.466$, 0.386 , and 0.411 , respectively). In the association of Model $1+$ APACHE II, Model $2+$ APACHE II, and Model $3+$ APACHE II, only the first was different from APACHE II ( $P=0.049,0.118$, and 0.125 , respectively). Table 3 shows the involved variables.

Using the variables related to the end point ( $\mathrm{HR}, \Delta T, \mathrm{CT}$, augmented index [AI], surgical, and APACHE II), a model was developed based on the ANN. At first, the network was trained and tested without the APACHE II variable, obtaining the degree of importance related to the following variables: $\Delta T(100 \%)$, HR (63.5\%), surgical (47.6\%), 
A
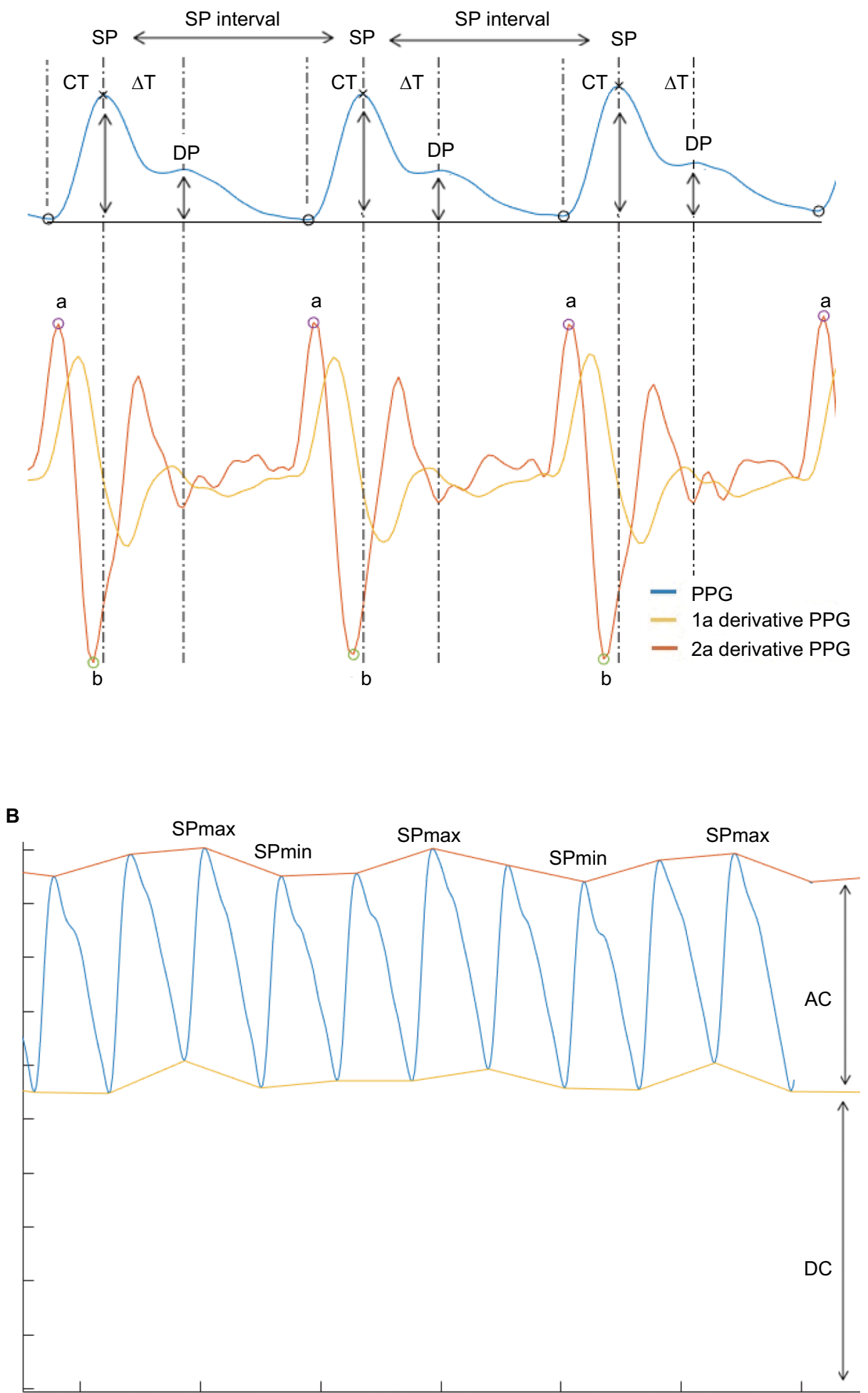

Figure I Identification of PPG parameters.

Abbreviations: SP, systolic peak; DP, diastolic peak; CT, crest time; SPmax, maximum systolic peak, SPmin, minimum systolic peak; AC, pulsatile component; DC, nonpulsatile component. 
Table I Characteristics of the ICC sample of HNSC in Tubarão, SC, Brazil

\begin{tabular}{ll}
\hline & $\mathbf{n}(\%)$ \\
\hline Age (years) & \\
$\geq 18$ and $<40$ & $24(12.6)$ \\
$\geq 40$ and $<60$ & $44(23.2)$ \\
$\geq 60$ & $122(64.2)$ \\
Gender & \\
Male & $106(55.8)$ \\
Female & $84(44.2)$ \\
Surgical & \\
No & $95(50.0)$ \\
Yes & $95(50.0)$ \\
Diagnosis & \\
Cardiovascular & $68(35.8)$ \\
Neurological & $39(20.5)$ \\
Gastrointestinal & $19(10.0)$ \\
Neoplasm & $18(9.5)$ \\
Respiratory & $14(7.4)$ \\
Infection & $13(6.8)$ \\
Polytrauma & $1 \mathrm{I}(5.8)$ \\
Orthopedic & $3(1.6)$ \\
Endocrine & $3(1.6)$ \\
Genitourinary & $2(1.1)$ \\
APACHE II & $14.0(8.0-19.0)$ \\
Probability of death (\%) & $18.6(8.7-32.2)$ \\
Length of ICC stay (days) & a \\
Death & $6.0(3.0-15.0)$ \\
No & \\
Yes & $136(71.6)$ \\
\hline Note & $54(28.4)$ \\
\hline
\end{tabular}

Note: amedian (percentile 25 - percentile 75).

Abbreviations: APACHE II, Acute Physiology and Chronic Health Evaluation; HNSC, Hospital Nossa Senhora da Conceição; ICC, intensive care center.
AI (37.8\%), and CT (26\%). In the comparison of ANN model with APACHE II, there was no statistical difference $(P=0.377)$. The variable APACHE II was then included in the ANN. The related degree of importance obtained was APACHE II (100\%), HR (68\%), $\Delta T$ (56.8\%), AI (54.4\%), surgical (51.8\%), and CT (35.6\%). In the association of ANN model + APACHE II, there was a statistical difference when compared with APACHE II $(P=0.027)$. We can see that the ANN accuracy was slightly higher than the previous models, performed by means of multivariate logistic regression (Table 4).

\section{Discussion}

The profile of the sample of this study showed a prevalence of male individuals (6:5) with a median age of 67 years, and the main reasons for hospitalization were cardiovascular and neurological causes. Half of them were surgical cases, and the median time of hospitalization was little more than a week. Death occurred in approximately one-fourth of the sample. Such data are similar to the study carried out in the ICU of Hospital das Clínicas (general hospital) of the Medical School of Marília, Brazil. ${ }^{21}$ A similar mortality rate, of nearly $20 \%$, was also found in a multicenter trial in European ICUs. ${ }^{22}$ However, it is different from the study carried out by Siddiqui, ${ }^{23}$ in Singapore, which showed a mortality rate of nearly $8 \%$ and the main reason for hospitalization was
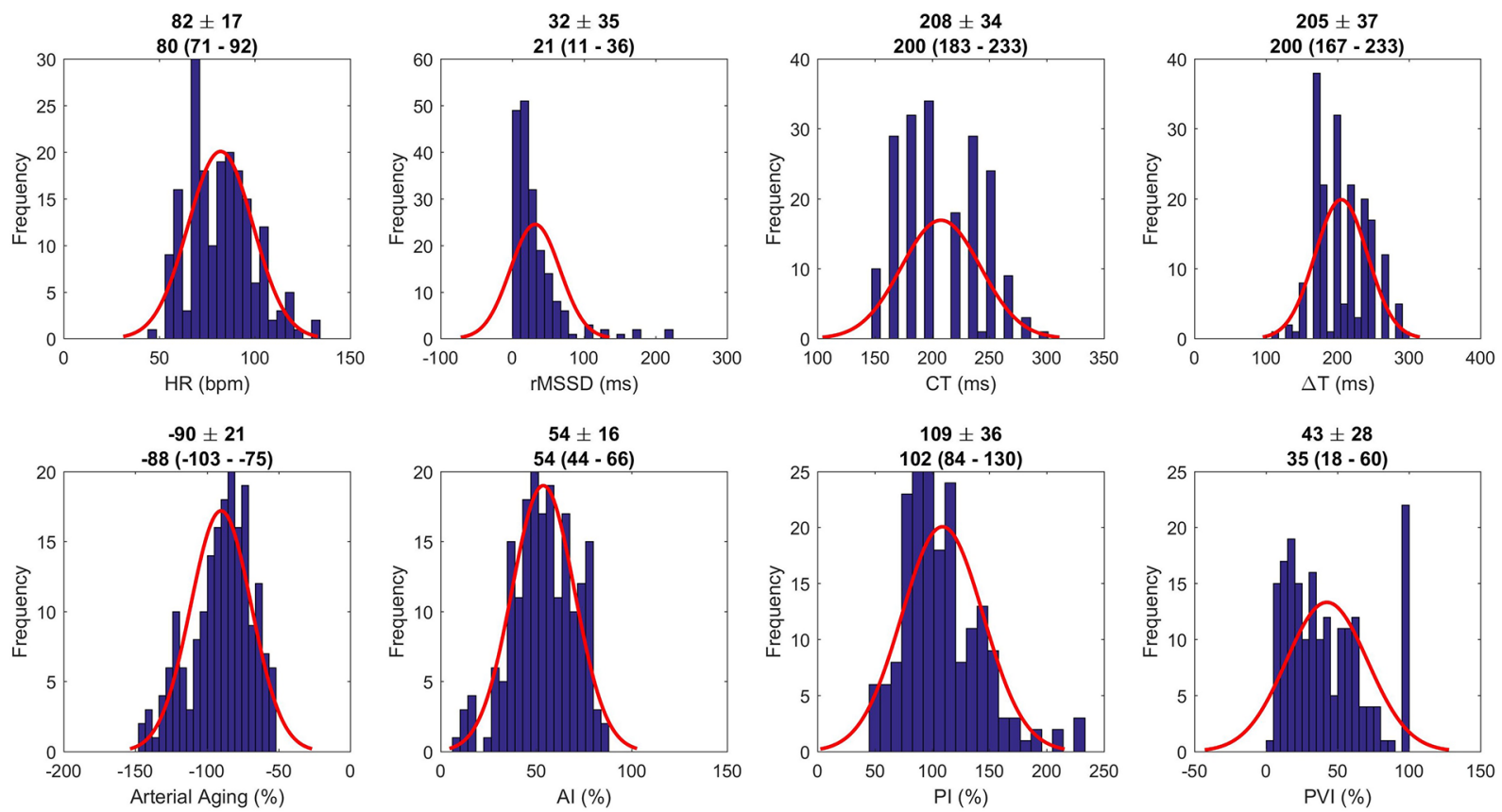

Figure 2 Variables related to PPG of the ICC sample of HNSC in Tubarão, SC, Brazil.

Abbreviations: $\Delta T$, time between the systolic and diastolic peaks; Al, augmented index; CT, crest time; HNSC, Hospital Nossa Senhora da Conceição; HR, heart rate; PI, perfusion index; PPG, Photoplethysmography; PVI, pleth variability index; rMSSD, root mean square of the successive differences. 
Table 2 Bivariate analysis and crude OR for death of the ICC sample of HNSC in Tubarão, SC, Brazil

\begin{tabular}{|c|c|c|c|c|}
\hline & High (median \pm IQL) & Death (median \pm IQL) & OR (95\% Cl) & $P$-value \\
\hline Age (years) & $65.5 \pm 21.0$ & $70.0 \pm 21.0$ & $1.023(1.001-1.044)$ & 0.039 \\
\hline \multicolumn{5}{|l|}{ Gender $^{\mathrm{a}}$} \\
\hline Male & $79(74.5)$ & $27(25.5)$ & 1.000 & \\
\hline Female & 57 (67.9) & $27(32.1)$ & $1.386(0.736-2.610)$ & 0.312 \\
\hline \multicolumn{5}{|l|}{ Surgical ${ }^{\mathrm{a}}$} \\
\hline Yes & $83(87.4)$ & $12(12.6)$ & 1.000 & \\
\hline No & $53(55.8)$ & $42(44.2)$ & $5.48 \mathrm{I}(2.646-1 \mathrm{I} .354)$ & $<0.001$ \\
\hline APACHE II & $11.5 \pm 10.0$ & $18.5 \pm 11.0$ & $1.168(1.106-1.234)$ & $<0.001$ \\
\hline Length of ICC stay (days) & $4.0 \pm 8.0$ & $10.5 \pm 16.0$ & 1.049 (1.02I-1.079) & 0.001 \\
\hline HR (bpm) & $80.0 \pm 23.1$ & $85.7 \pm 25.3$ & $1.020(1.001-1.039)$ & 0.036 \\
\hline rMSSD (ms) & $21.6 \pm 23.3$ & $19.3 \pm 36.6$ & $1.006(0.998-1.015)$ & 0.146 \\
\hline $\mathrm{CT}(\mathrm{ms})$ & $200.0 \pm 50.0$ & $200.0 \pm 50.0$ & $0.997(0.988-1.006)$ & 0.490 \\
\hline$\Delta T(\mathrm{~ms})$ & $200.0 \pm 50.0$ & $187.5 \pm 50.0$ & $0.989(0.979-0.998)$ & 0.015 \\
\hline Arterial aging (\%) & $-88.5 \pm 30.8$ & $-86.8 \pm 23.5$ & $1.008(0.093-1.023)$ & 0.316 \\
\hline $\mathrm{Al}(\%)$ & $52.7 \pm 22.0$ & $54.7 \pm 59.0$ & $1.000(0.991-1.008)$ & 0.922 \\
\hline PI (\%) & $102.7 \pm 45.4$ & $102.5 \pm 52.0$ & $1.027(0.423-2.497)$ & 0.952 \\
\hline PVI (\%) & $36.7 \pm 39.4$ & $34.1 \pm 44.9$ & $0.997(0.986-1.008)$ & 0.622 \\
\hline
\end{tabular}

Note: a Categorical variables expressed in $\mathrm{n}(\%)$.

Abbreviations: $\Delta T$, time between the systolic and diastolic peaks; Al, augmented index; APACHE II, Acute Physiology and Chronic Health Evaluation; CT, crest time; HNSC, Hospital Nossa Senhora da Conceição; HR, heart rate; ICC, intensive care center; IQL, interquartile; PI, perfusion index; PVI, pleth variability index; rMSSD, root mean square of the successive differences.

Table 3 Mortality prediction model based on epidemiological variables and PPG signal

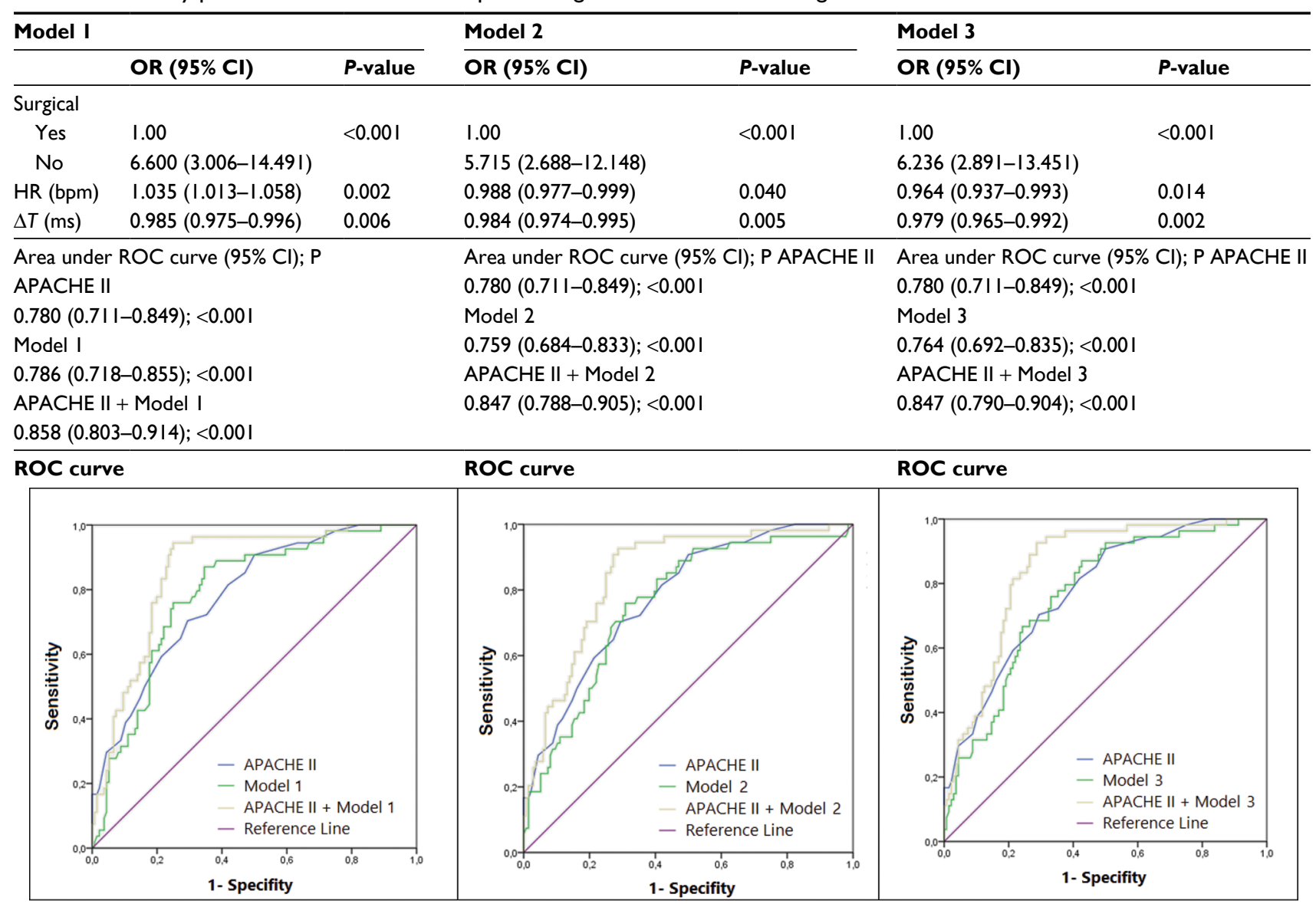

Abbreviations: $\Delta T$, time between the systolic and diastolic peaks; APACHE II, Acute Physiology and Chronic Health Evaluation; Al, augmented index; CT, crest time; HR, heart rate; PPG, Photoplethysmography; ROC, receiver-operating characteristic. 
Table 4 Prediction of mortality based on epidemiological variables and PPG signal, using ANNs

\section{ANNs without APACHE II}

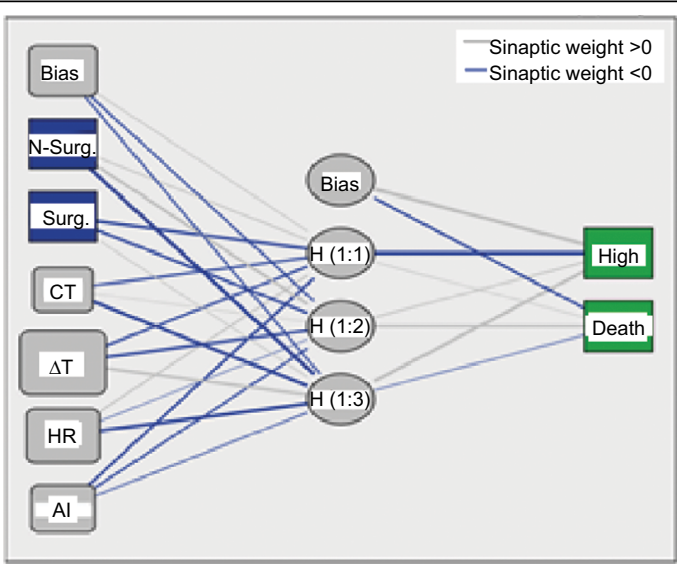

\begin{tabular}{llll} 
& \multicolumn{3}{l}{ Hidden layer } \\
\cline { 2 - 4 } & $\mathbf{H}(\mathbf{I}: \mathbf{I})$ & $\mathbf{H}(\mathbf{I}: 2)$ & $\mathbf{H}(\mathbf{I}: 3)$ \\
\hline (Bias) & 0.175 & -0.284 & -0.249 \\
Nonsurgical & 0.215 & 0.89 & -0.984 \\
Surgical & $-0.6 I I$ & -0.537 & 158 \\
CT & -0.347 & 0.039 & -0.700 \\
$\Delta T$ & -0.393 & -0.573 & 0.622 \\
HR & 0.244 & -0.065 & -0.806 \\
Al & -0.476 & -0.304 & $-0.19 \mathrm{I}$
\end{tabular}

Output layer

(Bias)

$\mathrm{H}(\mathrm{I}: \mathrm{I})$

$\mathrm{H}(\mathrm{I}: 2)$

$\mathrm{H}(\mathrm{I}: 3)$

$0.850-0.156$

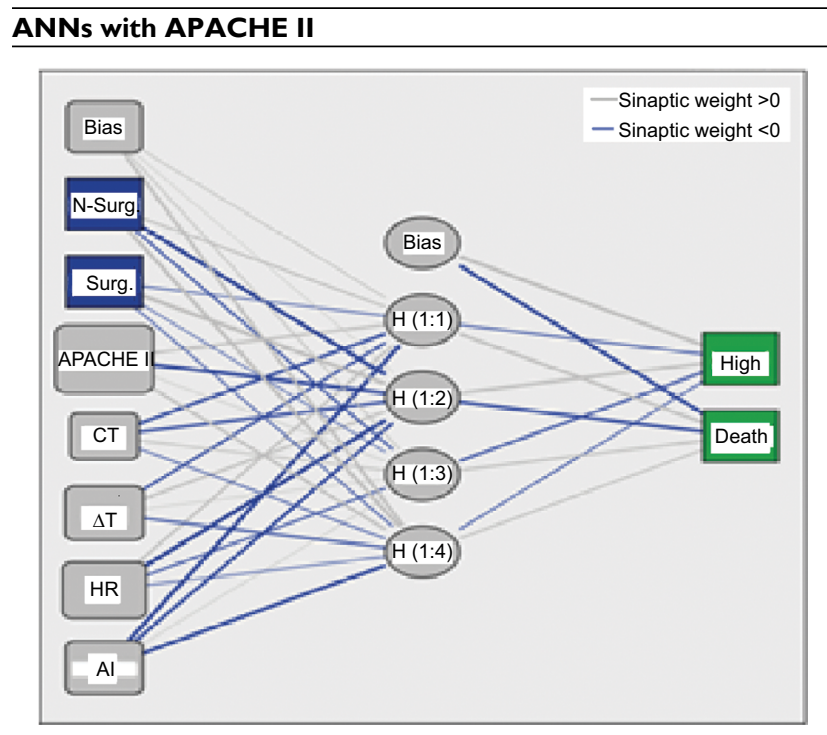

\begin{tabular}{|c|c|c|c|c|c|c|}
\hline & \multicolumn{4}{|c|}{ Hidden layer } & \multicolumn{2}{|c|}{ Output layer } \\
\hline & $(1: 1)$ & $\mathbf{H}(\mathrm{I}: 2)$ & $\mathbf{H}(\mathrm{I}: 3)$ & $H(I: 4)$ & High & Death \\
\hline (Bias) & 0.324 & 0.039 & 0.327 & I.297 & & \\
\hline Nonsurgical & 0.685 & -1.726 & -0.492 & 1.365 & & \\
\hline Surgical & -0.191 & 2.132 & -0.129 & $\begin{array}{l}-0.2 \\
\mathrm{I}\end{array}$ & & \\
\hline APACHE II & 0.866 & -1.449 & 0.124 & 0.992 & & \\
\hline CT & -1.080 & -0.814 & 0.473 & -0.283 & & \\
\hline$\Delta T$ & -0.709 & $0.81 \mathrm{I}$ & 0.070 & -0.620 & & \\
\hline $\mathrm{HR}$ & 0.780 & -2.004 & -0.452 & -0.179 & & \\
\hline $\mathrm{Al}$ & -1.514 & -1.110 & 0.076 & -1.363 & & \\
\hline (Bias) & & & & & $1.86 \mathrm{I}$ & -1.359 \\
\hline $\mathrm{H}(\mathrm{I}: \mathrm{I})$ & & & & & -0.452 & 1.254 \\
\hline $\mathrm{H}(\mathrm{I}: 2)$ & & & & & 1.324 & -1.140 \\
\hline $\mathrm{H}(\mathrm{I}: 3)$ & & & & & -0.640 & 0.745 \\
\hline $\mathrm{H}(\mathrm{I}: 4)$ & & & & & -0.312 & $\begin{array}{l}0.6 \\
1\end{array}$ \\
\hline
\end{tabular}

\section{ROC curve}

Area under ROC curve $(95 \% \mathrm{Cl})-P$

APACHE II

$0.780(0.711-0.849)-<0.001$

ANN model

$0.800(0.73 \mathrm{I}-0.868)-<0.00 \mathrm{I}$

APACHE II + ANN model $0.895(0.85 I-0.940)-<0.001$

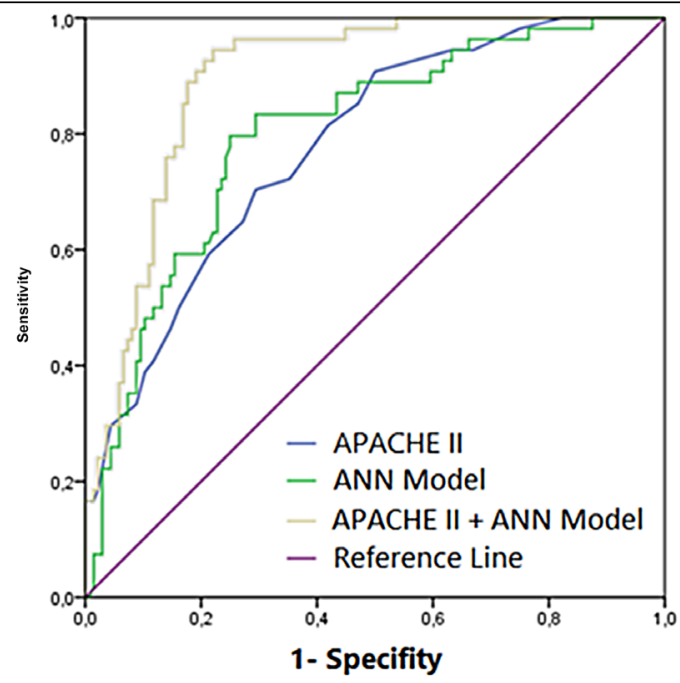

Abbreviations: APACHE II, Acute Physiology and Chronic Health Evaluation; Al, augmented index; ANNs, artificial neural networks; CT, crest time; PPG, Photoplethysmography; HR, heart rate; ROC, receiver-operating characteristic. 
septic shock, followed by neurological and cardiovascular causes. Moreover, a study carried out in an ICU in Nigeria identified a mortality rate above $60 \%$ and the neurological cases as main basic diagnoses. ${ }^{24}$ Such data show that the epidemiological profile of ICUs can change according to the area and structure of services.

With regard to the epidemiological aspects related to mortality, it was observed in this research that older individuals, nonsurgical cases, and longer periods of hospitalization were associated to death. The gender was not related to higher odds of dying. Although the review study conducted by Vezzani et $\mathrm{al}^{25}$ describes that, even with a higher number of male patients in the ICU, there is no difference in the end point between men and women. However, other studies ${ }^{26,27}$ found that, for uncertain reasons, women have higher odds of dying. On the other hand, advanced age, nonsurgical cases, and longer length of stay are undoubtedly described as factors related to higher odds of dying. ${ }^{28,29}$

The probability of death indicated by the APACHE II score was close to the probability found in this research, and the score was related to higher odds of dying, indicating that for each increase in the score, there is a corresponding increase in the risk of mortality of $\sim 17 \%$. Although the APACHE score is widely used as a prognostic measure and comparison of the severity of the disease among groups of patients, there are criticisms about its use, mainly because it does not consider the clinical evolution of the patient, requires careful attention in the counting of the points and is not suitable for subgroups of patients. For these reasons, it is indicated the complementation of other scores and the customization of existing scores. ${ }^{30,31}$

In this perspective, this study used the PPG to develop prediction models based on the pulse curve signal. One of the most basic parameters obtained with PPG is the HR. In this research, it was observed that tachycardia in the first day of hospitalization was related to the death, representing a $2 \%$ chance for each increase in heartbeat. This result was also found by Kara et al, ${ }^{32}$ who identified a higher risk of mortality in patients with HR $>100 / \mathrm{min}$ in the first 24 hours of hospitalization in the ICU. For specific cases, such as individuals with cerebrovascular accident, an HR little higher than 80 per minute in hospital admission is a marker of death risk. ${ }^{33,34}$ Such findings confirm this study, showing that perhaps the imbalance of the autonomic nervous system is apparent at an early stage in critically ill patients.

Even more specifically, the HR variability (HRV) is one of the measures that have been most used for analysis of the autonomic nervous system modulation. In ICUs, the major studies have focused on the use of HRV for the prediction of morbimortality. ${ }^{35}$ It is traditionally obtained by comparing the $\mathrm{R}-\mathrm{R}$ intervals in the electrocardiogram, differently from this study, which used the interval between the PPG SPs. Studies have shown that the analysis of the HRV is reliable in individuals at rest and that motion artifacts can contaminate the signal. ${ }^{36,37}$ In this study, this measure was taken excluding the ectopic beats and signal noises, for only 1 minute. This ultrashort time, made available by the PPG device manufacturer, allowed only the rMSSD measure analysis, which is very accurate when compared with the 5-minute measures. ${ }^{38}$ The results did not present a significant difference between the groups, as it was expected and shown in other studies, in which a lower HRV is related to a higher sympathovagal imbalance and higher mortality. ${ }^{35}$ Perhaps, the very short time in collecting data has influenced such results.

Regarding the PPG pulse wave form, only the $\Delta T$ parameter was individually associated to the death. For each decrease of 1 millisecond in the $\Delta T$, there is an increase of $1.1 \%$ in the odds of dying. This measure corresponds to the time between the SP and DP and is related to arterial stiffness. Physiologically, it indicates the time between the ejection of blood into the aorta and the reflection by the peripheral arterioles. This index is compared with the pulse wave velocity (PWV) when normalized by the individual's height. ${ }^{39}$ In this study, only $\Delta T$ was analyzed. From the epidemiological point of view, the arterial stiffness indicated by a higher PWV is related to mortality for all causes, as shown in the systematic review and meta analysis by Vlachopoulos et al. ${ }^{40}$ The results obtained in this research confirm such fact.

The other parameters of PPG pulse wave form, such as $\mathrm{AI}, \mathrm{CT}$, and b/a, do not show a statistical difference when individually compared with the group discharge and death. The AI corresponds to the height proportion between the DP and SP. It has an inverse correlation to the HR and is positively associated with peripheral vascular resistance, being subject to reduction in the infusion of nitroglycerin, a potent vasodilator. ${ }^{9}$ The $\mathrm{CT}$ is the time of ejection of blood into the aorta, after the cardiac isometric contraction and the SP, almost at the end of the systole. Its reduced value is a marker of cardiac dysfunction. ${ }^{41}$ The b/a index represents the stiffness of the large arteries, corresponding to the vascular response to the ejection of blood of the left ventricle. It has a positive correlation to arterial aging. ${ }^{10}$

In this study, the perfusion index did not show a statistical difference when compared with the group discharge and death. Many researches have shown the use of this measure as an important monitoring of the noninvasive peripheral perfusion. ${ }^{11,42}$ The research conducted by Pirneskoski et $\mathrm{al}^{5}$ showed that lower PI values are related to the most severe cases, serving as a tool for screening in emergency services. 
In the study conducted by Acar et al, ${ }^{43}$ the PI was monitored daily in the ICU. The authors showed that, only in the last day of hospitalization, the PI was able to stratify the death risk. And in the study conducted by Oskay et al, ${ }^{44}$ the prognostic capacity of this index in the emergency department was analyzed in more than 700 patients. The researchers did not find any association between the PI and admission in the ICU and hospital mortality. Likewise, in this study, the PI was not able to predict the risk of mortality in the first 24 hours of hospitalization. It is assumed that this measure is the most indicated in specific patient profiles, in a dynamic way, as follow-up in cases suggesting organic hypoperfusion, and it does not have much applicability in profiles of heterogeneous patients, as in this research.

The PVI parameter is derived from the PI variability and is related to hypovolemia. Its monitoring is important in patients who need volemic resuscitation, in which high indices indicate a greater need for volume replacement. ${ }^{4}$ The PVI is highly accurate only in mechanically ventilated patients. ${ }^{45}$ The research by Pirneskoski et $\mathrm{al}^{5}$ showed that higher PVI values can identify the cases with a greater need for intensive care, when evaluated in the emergency. However, in this study, we did not find any association between this variable and mortality. It is considered that, perhaps, the PVI is not adequate in the evaluation of patients in spontaneous breathing and without clinical correlation to the volemic state.

In the multivariate analysis, three models were proposed using the epidemiological surgical $\times$ nonsurgical variable and variables obtained with the PPG. Both models resulted in an accuracy close to the one obtained with APACHE II. Nonsurgical patients with arterial stiffness, identified with lower $\Delta T$ and with tachycardia in the first 24 hours of hospitalization generally have a higher death risk. The lower $\mathrm{CT}$ and AI, found in the multivariate analysis of Models 2 and 3 , seem to be related to cardiovascular dysfunction and reduction in the peripheral vascular resistance. Complementing the models to APACHE II, a greater capacity to predict mortality in the three cases was observed, showing that the combination of scores can improve the prognosis of patients hospitalized in ICUs. Moreover, with the use of a nonlinear algorithm, obtained by ANNs, it was observed that the end point prediction was more accurate, indicating that this form of analysis is an important tool for clinical judgment, as already presented in other studies. ${ }^{46,47}$

As limitations to the study, we can describe the heterogeneity of the sample, which may have made the convergence of the results difficult, the use of the PPG signal of only 1 minute, which made a more rigorous analysis of the HRV impossible, the sample loss for errors in the medical record identification, and poor quality of the signal, reducing the statistical power of data treatment.

\section{Conclusion}

This research analyzed the capacity to predict mortality in patients hospitalized in ICUs, by means of the PPG pulse curve. When compared with APACHE II, the accuracy was similar, but with the association of the proposed models with the APACHE II score, better results were observed. The use of ANNs was even more accurate, indicating that this tool is important to help in the clinical judgment of the intensivist.

However, more studies are necessary to compare such results with other ICU profiles.

\section{Author contributions}

All authors contributed toward data analysis, drafting and critically revising the article, gave final approval of the version to be published, and agree to be accountable for all aspects of the work.

\section{Disclosure}

The authors report no conflicts of interest in this work.

\section{References}

1. Golzari SE, Mahmoodpoor A. Care bundles in intensive care units. Lancet Infect Dis. 2014;14(5):371-372.

2. Rapsang AG, Shyam DC. Scoring systems in the intensive care unit: a compendium. Indian J Crit Care Med. 2014;18(4):220-228.

3. Vincent JL, Moreno R. Clinical review: scoring systems in the critically ill. Crit Care. 2010;14(2):207.

4. Nilsson LM. Respiration signals from photoplethysmography. Anesth Analg. 2013;117(4):859-865.

5. Pirneskoski J, Harjola VP, Jeskanen P, Linnamurto L, Saikko S, Nurmi J. Critically ill patients in emergency department may be characterized by low amplitude and high variability of amplitude of pulse photoplethysmography. Scand J Trauma Resusc Emerg Med. 2013;21:48.

6. Muhadi, M, Nasution SA, Putranto R, Harimurti K. The ability of detecting heart rate variability with the photoplethysmography to predict major adverse cardiac event in acute coronary syndrome. Acta Med Indones. 2016;48(1):48-53.

7. Elgendi M. On the analysis of fingertip photoplethysmogram signals Curr Cardiol Rev. 2012;8(1):14-25

8. Kohjitani A, Miyata M, Iwase Y, et al. Associations between the autonomic nervous system and the second derivative of the finger photoplethysmogram indices. J Atheroscler Thromb. 2014;21(5):501-508.

9. Millasseau SC, Kelly RP, Ritter JM, Chowienczyk PJ. Determination of age-related increases in large artery stiffness by digital pulse contour analysis. Clin Sci (Lond). 2002;103(4):371-377.

10. Otsuka T, Kawada T, Katsumata M, Ibuki C. Utility of second derivative of the finger photoplethysmogram for the estimation of the risk of coronary heart disease in the general population. Circ J. 2006;70(3):304-310.

11. Lima A. Bakker J. Noninvasive monitoring of peripheral perfusion. Intensive Care Med. 2005;31(10):1316-1326.

12. Broch O, Bein B, Gruenewald M, et al. Accuracy of the pleth variability index to predict fluid responsiveness depends on the perfusion index. Acta Anaesthesiol Scand. 2011;55(6):686-693.

13. Gandhi PG, Rao GH. The spectral analysis of photoplethysmography to evaluate an independent cardiovascular risk factor. Int J Gen Med. 2014;7:539-547. 
14. Trujillano J, March J, Sorribas A. Methodological approach to the use of artificial neural networks for predicting results in medicine. Med Clin (Barc). 2004;122(Suppl 1):59-67.

15. Clermont G, Angus DC, DiRusso SM, Griffin M, Linde-Zwirble WT. Predicting hospital mortality for patients in the intensive care unit: a comparison of artificial neural networks with logistic regression models. Crit Care Med. 2001;29(2):291-296.

16. Hajian-Tilaki K. Sample size estimation in diagnostic test studies of biomedical informatics. J Biomed Inform. 2014;48:193-204.

17. Knaus WA, Draper EA, Wagner DP, Zimmerman JE. APACHE II: a severity of disease classification system. Crit Care Med. 1985;13(10):818-829.

18. Jeyhani V, Mahdiani S, Peltokangas M, Vehkaoja A. Comparison of HRV parameters derived from photoplethysmography and electrocardiography signals. Conf Proc IEEE Eng Med Biol Soc. 2015;2015:5952-5965.

19. Rau HH, Hsu CY, Lin YA, et al. Development of a web-based liver cancer prediction model for type II diabetes patients by using an artificial neural network. Comput Methods Programs Biomed. 2016;125:58-65.

20. DeLong ER, DeLong DM, Clarke-Pearson DL. Comparing the areas under two or more correlated receiver operating characteristic curves: a nonparametric approach. Biometrics. 1988;44(3):837-845.

21. El-Fakhouri S, Carrasco HV, Araújo GC, Frini IC. Epidemiological profile of ICU patients at Faculdade de Medicina de Marília. Rev Assoc Med Bras (1992). 2016;62(3):248-254.

22. Capuzzo M, Volta CA, Tassinati T, et al; Working Group on Health Economics of the European Society of Intensive Care Medicine. Hospital mortality of adults admitted to intensive care units in hospitals with and without intermediate care units: a multicentre European cohort study. Crit Care. 2014;18(5):551.

23. Siddiqui S. Mortality profile across our intensive care units: a 5-year database report from a Singapore restructured hospital. Indian J Crit Care Med. 2015;19(12):726-727.

24. Iwuafor AA, Ogunsola FT, Oladele RO, et al. Incidence, clinical outcome and risk factors of intensive care unit infections in the Lagos University Teaching Hospital (LUTH), Lagos, Nigeria. PLoS ONE. 2016;11(10):e0165242.

25. Vezzani A, Manca C, Ermio C. Gender disparities in the intensive care unit. Ital J Gender Specific Med. 2016;2(1):22-27

26. Lipes J, Mardini L, Jayaraman D. Sex and mortality of hospitalized adults after admission to an intensive care unit. Am J Crit Care. 2013;22(4): 314-319.

27. Mahmood K, Eldeirawi K, Wahidi MM. Association of gender with outcomes in critically ill patients. Crit Care. 2012;16(3):R92.

28. Colpan A, Akinci E, Erbay A, Balaban N, Bodur H. Evaluation of risk factors for mortality in intensive care units: a prospective study from a referral hospital in Turkey. Am J Infect Control. 2005;33(1):42-7.

29. Santana-Cabrera L, Lorenzo-Torrenta R, Sánchez-Palaciosa M, Martín Santanab JD, Hernández Hernándezb JR. Influencia de la edad en la duración de la estancia y en la mortalidad de los pacientes que permanecen de forma prolongada en una Unidad de Cuidados Intensivo. Revista Clínica Española (English Edition). 2014;214(2):74-78.

30. Salluh JI, Soares M. ICU severity of illness scores: APACHE, SAPS and MPM. Curr Opin Crit Care. 2014;20(5):557-565.

31. Ferrando-Vivas P, Jones A, Rowan KM, Harrison DA. Development and validation of the new ICNARC model for prediction of acute hospital mortality in adult critical care. J Crit Care. 2017;38:335-339.
32. Kara D, Akinci SB, Babaoglu G, Aypar U. Increased heart rate on first day in intensive care unit is associated with increased mortality. PakJ Med Sci. 2016;32(6):1402-1407.

33. Erdur H, Grittner U, Scheitz JF, Laufs U, Endres M, Nolte CH. Heart rate on admission independently predicts in-hospital mortality in acute ischemic stroke patients. Int J Cardiol. 2014;176(1):206-210.

34. Nolte $\mathrm{CH}$, Erdur $\mathrm{H}$, Grittner U, et al. Impact of heart rate on admission on mortality and morbidity in acute ischaemic stroke patients - results from VISTA. Eur J Neurol. 2016;23(12):1750-1756.

35. Karmali SN, Sciusco A, May SM, Ackland GL. Heart rate variability in critical care medicine: a systematic review. Intensive Care Med Exp. 2017;5(1):33.

36. Lu G, Yang F, Taylor JA, Stein JF. A comparison of photoplethysmography and ECG recording to analyse heart rate variability in healthy subjects. J Med Eng Technol. 2009;33(8):634-641.

37. Schäfer A, Vagedes J. How accurate is pulse rate variability as an estimate of heart rate variability? A review on studies comparing photoplethysmographic technology with an electrocardiogram. Int $J$ Cardiol. 2013;166(1):15-29.

38. Baek HJ, Cho CH, Cho J, Woo JM. Reliability of ultra-short-term analysis as a surrogate of standard 5-min analysis of heart rate variability. Telemed J E Health. 2015;21(5):404-414.

39. Millasseau SC, Ritter JM, Takazawa K, Chowienczyk PJ. Contour analysis of the photoplethysmographic pulse measured at the finger. J Hypertens. 2006;24(8):1449-1456.

40. Vlachopoulos C, Aznaouridis K, Stefanadis C. Prediction of cardiovascular events and all-cause mortality with arterial stiffness: a systematic review and meta-analysis. J Am Coll Cardiol. 2010;55(13): 1318-1327.

41. Biering-Sørensen T, Querejeta Roca G, Hegde SM, et al. Left ventricular ejection time is an independent predictor of incident heart failure in a community-based cohort. Eur J Heart Fail. 2018;20(7): 1106-1114.

42. He H, Long Y, Liu D, Wang X, Zhou X. Clinical classification of tissue perfusion based on the central venous oxygen saturation and the peripheral perfusion index. Crit Care. 2015;19:330.

43. Acar Y, Yamanel L, Cinar O, Cevik E, Kilic S, Yasar M. Perfusion index from pulse oximetry predicts mortality and correlates with illness severity scores in intensive care unit patients. Acta Medica Mediterranea. 2015;31:237-242.

44. Oskay A, Eray O, Dinç SE, Aydın AG, Eken C. Prognosis of critically ill patients in the ED and value of perfusion index measurement: a cross-sectional study. Am J Emerg Med. 2015;33(8):1042-1044.

45. Sandroni C, Cavallaro F, Marano C, Falcone C, De Santis P, Antonelli $\mathrm{M}$. Accuracy of plethysmographic indices as predictors of fluid responsiveness in mechanically ventilated adults: a systematic review and meta-analysis. Intensive Care Med. 2012;38(9):1429-1437.

46. LaFaro RJ, Pothula S, Kubal KP, et al. Neural network prediction of ICU length of stay following cardiac surgery based on pre-incision variables. PLoS ONE. 2015;10(12):e0145395.

47. Gholipour C, Rahim F, Fakhree A, Ziapour B. Using an artificial neural networks (ANNs) model for prediction of intensive care unit (ICU) outcome and length of stay at hospital in traumatic patients. J Clin Diagn Res. 2015;9(4):OC19-OCT23.
Vascular Health and Risk Management

\section{Publish your work in this journal}

Vascular Health and Risk Management is an international, peerreviewed journal of therapeutics and risk management, focusing on concise rapid reporting of clinical studies on the processes involved in the maintenance of vascular health; the monitoring, prevention and treatment of vascular disease and its sequelae; and the involvement of
Dovepress

metabolic disorders, particularly diabetes. This journal is indexed on PubMed Central and MedLine. The manuscript management system is completely online and includes a very quick and fair peer-review system, which is all easy to use. Visit http://www.dovepress.com/ testimonials.php to read real quotes from published authors. 